\title{
THE CONVERGENCE OF RATIONAL FUNCTIONS OF BEST APPROXIMATION TO THE EXPONENTIAL FUNCTION. II
}

\author{
E. B. $S A F F^{1}$
}

\begin{abstract}
Let $W_{m, n}(z)$ be a rational function of type $(m, n)$ of best uniform approximation to the function $e^{z}$ on the closed unit disk. In this paper we show that any sequence $\left\{\boldsymbol{W}_{m, n}(z)\right\}$ for which $m+n \rightarrow \infty$ must converge to $e^{z}$ for all values of $z$. This is the first result which describes completely the regions of convergence of arbitrary sequences formed from a Walsh array.
\end{abstract}

1. Introduction. A rational function $r_{m, n}(z)$ is said to be of type $(m, n)$ if it is of the form

$$
r_{m, n}(z)=p_{m}(z) / q_{n}(z), \quad q_{n}(z) \not \equiv 0,
$$

where $p_{m}(z)$ is a polynomial of degree at most $m$ and $q_{n}(z)$ is a polynomial of degree at most $n$. For each pair $(m, n)$ let $W_{m, n}(z)$ denote a rational function of type $(m, n)$ of best uniform approximation to the function $e^{z}$ on the disk $|z| \leqq 1$. By "best uniform approximation" we mean that the inequality

$$
\max _{|z| \leqq 1}\left|e^{z}-W_{m, n}(z)\right| \leqq \max _{|z| \leqq 1}\left|e^{z}-r_{m, n}(z)\right|
$$

holds for every rational function $r_{m, n}(z)$ of type $(m, n)$. The $W_{m, n}(z)$ form a doubly-infinite array

$$
\begin{array}{cccc}
W_{0,0}(z) & W_{1,0}(z) & W_{2,0}(z) & \cdots \\
W_{0,1}(z) & W_{1,1}(z) & W_{2,1}(z) & \cdots \\
W_{0,2}(z) & W_{1,2}(z) & \cdots & \cdots
\end{array}
$$

known as the $L_{\infty}$ Walsh array [6] for $e^{z}$ on $|z| \leqq 1$.

Presented to the Society, September 14, 1970; received by the editors September 12, 1970.

AMS 1970 subject classifications. Primary 41A20, 41A50; Secondary 30A82, 15 A45. Key words and phrases. Best uniform approximation, rational functions of type $(m, n)$, Walsh array, Padé table, Bernstein lemma, Taylor development, polynomial of least squares approximation.

${ }^{1}$ The research of the author was supported, in part, by NSF Grant GF-19275.

(c) American Mathematical Society 1972 
The regions of convergence of the rows and columns of Walsh arrays for meromorphic functions have been investigated in [3] and [7]. However, no result is to be found in the literature which concerns the regions of convergence of other sequences of rational functions which may be formed from an array, such as the diagonals. The aim of the present paper is to establish such a convergence theorem for the array (1).

MAIN THEOREM. Let $W_{m, n}(z)$ be any sequence of rational functions from the array (1) such that $m+n \rightarrow \infty$. Then for all values of $z$ we have

$$
\lim _{m+n \rightarrow \infty} W_{m, n}(z)=e^{z}
$$

and the limit is uniform on each bounded subset of the plane.

In [4] the author proved (2) for the special cases where $m$ or $n$ is fixed, i.e., for the columns and rows of (1). The proofs of the special cases and of the general result depend upon a comparison of (1) with the Padé table [2] for $e^{z}$. The Padé approximant of type $(m, n)$ for the function $e^{z}$ is the unique rational function $R_{m, n}(z)$ of type $(m, n)$ with the property that $e^{z}-R_{m, n}(z)$ has a zero of order $m+n+1$ at $z=0$. We shall write

$$
R_{m, n}(z)=P_{m, n}(z) / Q_{m, n}(z),
$$

where $P_{m, n}(z)$ and $Q_{m, n}(z)$ are polynomials of respective degrees $m$ and $n$, and $Q_{m, n}(0)=1$. We call $P_{m, n}(z)$ a Padé numerator and $Q_{m, n}(z)$ a Padé denominator for the function $e^{z}$.

2. Lemmata. In this section we mention some results that will be used to prove (2).

LEMMA 1. Let $\tau>0$ and $R_{m, n}(z)$ a sequence of Padé approximants for $e^{z}$ such that $m+n \rightarrow \infty$. Then for $m+n$ sufficiently large we have

$$
\begin{aligned}
& \left|e^{z}-R_{m, n}(z)\right| \leqq A_{\tau} \tau^{m+n+1} m ! n ! /(m+n) !(m+n+1) !, \\
& |z| \leqq \tau .
\end{aligned}
$$

Furthermore, the Padé denominators $Q_{m, n}(z)$ for $e^{z}$ satisfy, for all $z$,

$$
\left|Q_{m, n}(z)-e^{-n z /(m+n)}\right| \leqq|z|^{2} e^{|z|} / 2(m+n), \quad m+n>0 .
$$

Here and below constants $A$ with subscripts are independent of $m$ and $n$.

Proof. Inequality (4) is proved in $[2, \S 75]$ and implies that for $m+n$ sufficiently large the $Q_{m, n}(z)$ are in modulus uniformly bounded below by a positive constant for $|z| \leqq \tau$. From the known identity [2, p. 436]

$$
e^{z} Q_{m, n}(z)-P_{m, n}(z)=\frac{(-1)^{n} z^{m+n+1}}{(m+n) !} \int_{0}^{1} e^{t z} t^{n}(1-t)^{m} d t
$$


we obtain

$$
\left|e^{z} Q_{m, n}(z)-P_{m, n}(z)\right| \leqq e^{\tau} \tau^{m+n+1} m ! n ! /(m+n) !(m+n+1) !,
$$

$|z| \leqq \tau$

and (3) follows.

The next lemma appears in [5].

LEMMA 2. Let $C^{n-1}$ denote the set of all ordered (n-1)-tuples $Z=$ $\left(z_{1}, z_{2}, \cdots, z_{n-1}\right)$ of complex numbers and for $k=1,2, \cdots, n$ let

$$
L_{k}(Z) \equiv b_{k}+\sum_{i=1}^{n-1} b_{k i} z_{i}
$$

Let $B$ denote the determinant of the matrix

$$
\left[\begin{array}{ccccc}
b_{1} & b_{11} & b_{12} & \cdots & b_{1, n-1} \\
b_{2} & b_{21} & b_{22} & \cdots & b_{2, n-1} \\
\cdot & \cdot & \cdot & & \cdot \\
\cdot & \cdot & \cdot & & \cdot \\
\cdot & \cdot & \cdot & & \cdot \\
b_{n} & b_{n 1} & b_{n 2} & \cdots & b_{n, n-1}
\end{array}\right]
$$

and for $1 \leqq \mu \leqq n$ let $B^{(\mu)}$ be the determinant of the matrix obtained by deleting the $\mu$ th row and 1 st column from the matrix $(5)$. If $B^{(\mu)} \neq 0$ for some $\mu$, then

$$
\sum_{k=1}^{n}\left|L_{k}(Z)\right| \geqq \min _{1 \leqq \mu \leqq n}\left\{|B| /\left|B^{(\mu)}\right|\right\}, \quad Z \in C^{n-1} .
$$

LEMMA 3. Let

(6)

$$
M^{m, n} \equiv \operatorname{det}\left[\begin{array}{cccc}
1 / m ! & 1 /(m-1) ! & \cdots & 1 /(m-n+1) ! \\
1 /(m+1) ! & 1 / m ! & \cdots & 1 /(m-n+2) ! \\
\cdot & & & \cdot \\
\cdot & & & \cdot \\
\cdot & & & \cdot \\
1 /(m+n-1) ! & 1 /(m+n-2) ! & \cdots & 1 / m !
\end{array}\right] \text {, }
$$

If $M_{\mu, v}^{m, n}$ denotes the determinant of the matrix obtained by deleting the $\mu$ th row and $v$ th column from the matrix in (6), then

$$
0<M_{\mu, \nu}^{m, n} \leqq M_{\mu, 1}^{m, n}, \quad 1 \leqq \mu \leqq n, \quad 1 \leqq \nu \leqq n .
$$

For $m-k<0$, we put $1 /(m-k) !=0$ in (6). 
Proof. The following identities are derived in [4] for $m \geqq n$, but remain valid without this restriction:

$$
\begin{gathered}
M^{m, n}=\prod_{i=1}^{n} \frac{(n-i) !}{(m-1+i) !}, \\
M_{\mu, 1}^{m, n}=\frac{1}{(\mu-1) !} \prod_{i=1 ; i \neq \mu}^{n} \frac{(n-i) !}{(m-2+i) !}, \quad M_{1, v}^{m, n}=M_{n-v+1,1}^{m+1, n} .
\end{gathered}
$$

The equations (8) imply

$$
M_{1, v}^{m, n} \leqq M_{1, v-1}^{m, n},
$$

and so (7) holds for $\mu=1$. For $\mu>1$ we prove (7) by induction on $\nu$.

By a well-known result of the theory of determinants [1, p. 33, Corollary 3]

$$
M_{\mu, \nu}^{m, n}=M_{\mu, 1}^{m, n}\left[\frac{M_{1, v}^{m, n}}{M_{1,1}^{m, n}}+\frac{M^{m, n} M_{\mu-1, v-1}^{m, n-1}}{M_{1,1}^{m, n} M_{\mu, 1}^{m, n}}\right], \quad \mu>1, \quad \nu>1 .
$$

Thus for $\nu=2$ we obtain, from (10) and the identities (8),

$$
M_{\mu, 2}^{m, n}=M_{\mu, 1}^{m, n}\left[\frac{(n-1)(m+\mu-2)+\mu-1}{(m+n-1)(m+\mu-2)}\right] \leqq M_{\mu, 1}^{m, n}, \quad \mu>1 .
$$

Now assume that (7) holds for $\nu=k(>1)$ so that $M_{\mu-1, k}^{m, n-1} \leqq M_{\mu-1,1}^{m, n-1}$. Since (9) implies that $M_{1, k+1}^{m, n} \leqq M_{1,2}^{m, n}$, we deduce, from (10),

$$
M_{\mu, k+1}^{m, n} \leqq M_{\mu, 2}^{m, n} \leqq M_{\mu, 1}^{m, n},
$$

i.e., (7) holds for $\nu=k+1$. This proves Lemma 3.

We shall use Lemmas 2 and 3 to establish

LEMMA 4. Let $\rho>0, m \geqq 1, n \geqq 2,0 \leqq i_{0} \leqq n-1$, and $z_{i_{0}} \equiv 1$. Then for any $n-1$ complex numbers $z_{i}, 0 \leqq i \leqq n-1, i \neq i_{0}$, we have

$$
\begin{aligned}
\sum_{k=1}^{n} \mid \sum_{i=0}^{n-1} z_{i} /(m+ & k-1-i) ! \mid \rho^{k} \\
& \geqq \lambda \rho^{n} \frac{(m-1) !(n-1) !}{(m+n-2) !(m+n-1) !},
\end{aligned}
$$

where $\lambda \equiv \inf _{0 \leqq j<\infty}\left\{j ! / \rho^{j}\right\}>0$.

Proof. Lemma 2 implies that the left-hand member of (11) is bounded from below by $\min _{1 \leqq \mu \leqq n}\left\{\rho^{\mu} M^{m, n} / M_{\mu, i_{0}+1}^{m, n}\right\}$, and hence, from (7), by $\min _{1 \leqq \mu \leqq n}\left\{\rho^{\mu} M^{m, n} / M_{\mu, 1}^{m, n}\right\}$. Since

$$
\frac{\rho^{\mu} M_{n, 1}^{m, n}}{\rho^{n} M_{\mu, 1}^{m, n}}=\frac{(n-\mu) !(\mu-1) !(m+n-2) !}{\rho^{n-\mu}(n-1) !(m+\mu-2) !} \geqq \frac{(n-\mu) !}{\rho^{n-\mu}} \geqq \lambda,
$$


we have

$$
\rho^{\mu} M^{m, n} / M_{\mu, 1}^{m, n} \geqq \lambda \rho^{n} M^{m, n} / M_{n, 1}^{m, n}=\lambda \rho^{n} \frac{(m-1) !(n-1) !}{(m+n-2) !(m+n-1) !},
$$

and (11) follows.

The final lemma is due to J. L. Walsh [8, p. 231].

LEMMA 5. If $r(z)$ is a rational function of type $(m, m)$ whose poles lie exterior to the circle $|z|=R(>1)$, and if $|r(z)| \leqq L$, for $|z| \leqq 1$, then

$$
|r(z)| \leqq L\left(\frac{R \tau-1}{R-\tau}\right)^{m}, \text { for }|z| \leqq \tau, \quad 1<\tau<R .
$$

3. Proof of Main Theorem. We first show that as $m+n \rightarrow \infty$ the poles of the $W_{m, n}(z)$ approach infinity. Suppose to the contrary that there exists a number $\rho>1$ and a subsequence of the $W_{m, n}(z)$, which we continue to denote by $W_{m, n}(z)$, with the property that each $W_{m, n}(z)$ has a pole $1 / \alpha_{m, n}\left(\left|\alpha_{m, n}\right|<1\right)$ that lies in the disk $|z| \leqq \rho / 2$. We assume, as we may, that $m \geqq 1$. Now write

$$
W_{m, n}(z)=p_{m, n}(z) /\left(1-\alpha_{m, n} z\right) q_{m, n}(z),
$$

where $p_{m, n}(z)$ is a polynomial of degree at most $m$, and $q_{m, n}(z)$ is a polynomial of the form

$$
q_{m, n}(z)=\sum_{i=0}^{n-1} c_{i}^{m, n} z^{i}
$$

which is normalized so that $\left|c_{i}^{m, n}\right| \leqq 1,0 \leqq i \leqq n-1$, and $c_{i_{0}}^{m, n}=1$ for some $i_{0}=i_{0}(m, n)$. By the extremal property of the $W_{m, n}(z)$ we have, from Lemma 1,

$$
\left|e^{z}-W_{m, n}(z)\right| \leqq A_{1} \varepsilon_{m, n}, \quad|z| \leqq 1,
$$

where $\varepsilon_{m, n} \equiv m ! n ! /(m+n) !(m+n+1)$ !. Thus by the triangle inequality

$$
\left|R_{m, n}(z)-W_{m, n}(z)\right| \leqq 2 A_{1} \varepsilon_{m, n}, \quad|z| \leqq 1 .
$$

Since (4) implies that the Padé denominators $Q_{m, n}(z)$ are uniformly bounded for $|z| \leqq 1$, we have

$$
\left|Q_{m, n}(z)\left(1-\alpha_{m, n} z\right) q_{m, n}(z)\right| \leqq A_{2} n, \quad|z| \leqq 1,
$$

and so from (13) there follows

$$
\left|\left(1-\alpha_{m, n} z\right) q_{m, n}(z) P_{m, n}(z)-Q_{m, n}(z) p_{m, n}(z)\right| \leqq A_{3} n \varepsilon_{m, n}, \quad|z| \leqq 1 .
$$

Note that the function whose absolute value appears in the last inequality is a polynomial of degree at most $m+n$ and hence from a lemma of 
Bernstein $[8$, p. 77$]$ we deduce that

$\left|\left(1-\alpha_{m, n} z\right) q_{m, n}(z) P_{m, n}(z)-Q_{m, n}(z) p_{m, n}(z)\right| \leqq A_{3} \rho^{m+n} n \varepsilon_{m, n}, \quad|z| \leqq \rho$.

But for $m+n$ sufficiently large $\left|Q_{m, n}(z)\right| \geqq \sigma>0$ for $|z| \leqq \rho$, and so

(14) $\left|\left(1-\alpha_{m, n} z\right) q_{m, n}(z) R_{m, n}(z)-p_{m, n}(z)\right| \leqq A_{4} \rho^{m+n} n \varepsilon_{m, n}, \quad|z| \leqq \rho$.

Since (14) holds for $z=1 / \alpha_{m, n}$, it follows that

$$
\begin{aligned}
& \qquad\left(1-\alpha_{m, n} z\right) q_{m, n}(z) R_{m, n}(z)-p_{m, n}(z)+p_{m, n}\left(1 / \alpha_{m, n}\right) \mid \leqq 2 A_{4} \rho^{m+n} n \varepsilon_{m, n}, \\
& \text { and consequently } \\
& \qquad|z| \leqq \rho,
\end{aligned}
$$

$$
\max _{|z| \leqq \rho}\left|q_{m, n}(z) R_{m, n}(z)-\pi_{m, n}(z)\right| \leqq A_{5} \rho^{m+n} n \varepsilon_{m, n},
$$

where $\pi_{m, n}(z) \equiv\left(p_{m, n}(z)-p_{m, n}\left(1 / \alpha_{m, n}\right)\right) /\left(1-\alpha_{m, n} z\right)$ is a polynomial of degree at most $m-1$.

We now obtain a lower bound for the left-hand member of (15). Let

$$
R_{m, n}(z)=\sum_{j=0}^{\infty} d_{j}^{m, n} z^{j}
$$

Since the $(m-1)$ th section of the Taylor development

$$
q_{m, n}(z) R_{m, n}(z)=\sum_{j=0}^{\infty}\left(\sum_{i=0}^{n-1} c_{i}^{m, n} d_{j-i}^{m, n}\right) z^{j}, \quad d_{j-i}^{m, n}=0 \quad \text { for } \quad j-i<0,
$$

is the polynomial of degree at most $m-1$ of least squares approximation to the function $q_{m, n}(z) R_{m, n}(z)$ on $|z|=\rho$, we have the inequalities

$$
\begin{aligned}
{\left[\max _{|z| \leqq \rho} \mid q_{m, n}(z)\right.} & \left.R_{m, n}(z)-\pi_{m, n}(z) \mid\right]^{2} \\
& \geqq(2 \pi \rho)^{-1} \int_{|z|=\rho}\left|q_{m, n}(z) R_{m, n}(z)-\pi_{m, n}(z)\right|^{2}|d z| \\
& \geqq(2 \pi \rho)^{-1} \int_{|z|=\rho}\left|\sum_{j=m}^{\infty}\left(\sum_{i=0}^{n-1} c_{i}^{m, n} d_{j-i}^{m, n}\right) z^{j}\right|^{2}|d z| \\
& \geqq \sum_{j=m}^{m+n-1}\left|\sum_{i=0}^{n-1} c_{i}^{m, n} d_{j-i}^{m, n}\right|^{2} \rho^{2 j} \\
& \geqq n^{-1}\left[\sum_{j=m}^{m+n-1}\left|\sum_{i=0}^{n-1} c_{i}^{m, n} d_{j-i}^{m, n}\right| \rho^{j}\right]^{2} ;
\end{aligned}
$$

the last inequality follows from the Cauchy-Schwarz inequality. By the 
definition of the $R_{m, n}(z)$,

$$
d_{j}^{m, n}=1 / j !, \text { for } 0 \leqq j \leqq m+n .
$$

Hence (16) implies that

$$
\begin{aligned}
& \max _{|z| \leqq \rho} \mid q_{m, n}(z) R_{m, n}(z)-\pi_{m, n}(z) \mid \\
& \geqq \frac{\rho^{m-1}}{\sqrt{ } n} \sum_{k=1}^{n}\left|\sum_{i=0}^{n-1} c_{i}^{m, n} /(m+k-1-i) !\right| \rho^{k},
\end{aligned}
$$

and so from Lemma 4 we obtain

$$
\max _{|z| \leqq \rho}\left|q_{m, n}(z) R_{m, n}(z)-\pi_{m, n}(z)\right| \geqq \frac{\lambda}{\sqrt{ } n} \rho^{m+n-1} \varepsilon_{m-1, n-1} .
$$

But then by (15) and (17),

$$
A_{5} \rho^{m+n} n \varepsilon_{m, n} \geqq \frac{\lambda}{\sqrt{ } n} \rho^{m+n-1} \varepsilon_{m-1, n-1},
$$

which contradicts the fact that $n^{3 / 2} \varepsilon_{m, n} / \varepsilon_{m-1, n-1} \rightarrow 0$ as $m+n \rightarrow \infty$. Thus the $W_{m, n}(z)$ have no finite limit point of poles.

We can now prove (2). Let $1<\tau<R$ and choose $m+n$ so large that the poles of $W_{m, n}(z)$ and of $R_{m, n}(z)$ lie exterior to the circle $|z|=R$. Since $R_{m, n}(z)-W_{m, n}(z)$ is a rational function of type $(2(m+n), 2(m+n))$ it follows from (13) and Lemma 5 that

$$
\left|R_{m, n}(z)-W_{m, n}(z)\right| \leqq 2 A_{1} \varepsilon_{m, n}\left(\frac{R \tau-1}{R-\tau}\right)^{2(m+n)}, \quad|z| \leqq \tau .
$$

Thus from (3) and the triangle inequality we obtain

(18) $\max _{|z| \leqq \tau}\left|e^{z}-W_{m, n}(z)\right| \leqq A_{\tau} \tau^{m+n+1} \varepsilon_{m, n}+2 A_{1} \varepsilon_{m, n}\left(\frac{R \tau-1}{R-\tau}\right)^{2(m+n)}$.

As $m+n \rightarrow \infty$ the right-hand member of (18) approaches zero and so (2) holds for $|z| \leqq \tau$. Since $\tau(>1)$ is arbitrary, the proof of the Main Theorem is complete.

We conclude with two remarks. Firstly, by applying the method used to obtain (17) it can be shown that

$$
\max _{|z| \leqq 1}\left|e^{z}-W_{m, n}(z)\right| \geqq \varepsilon_{m, n} /(n+1)^{3 / 2} .
$$

Secondly, the convergence in (2) holds not only for the extremal rational functions $W_{m, n}(z)$, but for any sequence of rational functions $W_{m, n}(z)$ of respective types $(m, n)$ which satisfy $(12)$. 


\section{REFERENCES}

1. M. Bôcher, Introduction to higher algebra, Macmillan, New York, 1907.

2. O. Perron, Die Lehre von den Kettencrüchen, 2nd ed., Chelsea, New York, 1929.

3. E. B. Saff, On the row convergence of the Walsh array for meromorphic functions, Trans. Amer. Math. Soc. 146 (1969), 241-257. MR 42 \#517.

4. - The convergence of rational functions of best approximation to the exponential function, Trans. Amer. Math. Soc. 153 (1971), 483-493.

5. - Regions of meromorphy determined by the degree of best rational approximation, Proc. Amer. Math. Soc. 29 (1971), 30-38.

6. J. L. Walsh, On approximation to an analytic function by rational functions of best approximation, Math. Z. 38 (1934), 163-176.

7. - The convergence of sequences of rational functions of best approximation with some free poles, Approximation of Functions (Proc. Sympos. General Motors Res. Lab., 1964), Elsevier, Amsterdam, 1965, pp. 1-16. MR 32 \#4441.

8. - Interpolation and approximation by rational functions in the complex domain, 5th ed., Amer. Math. Soc. Colloq. Publ., vol. 20, Amer. Math. Soc., Providence, R.I., 1969.

Department of Mathematics, University of South Florida, Tampa, Florida 33620 